\title{
Two new records in Pestalotiopsidaceae associated with Orchidaceae disease in Guangxi Province, China
}

\section{Ran $\mathrm{SF}^{1}$, Maharachchikumbura $\mathrm{SSN}^{2}$, Ren $\mathrm{YL}^{3}$, Liu $\mathrm{H}^{4}$, Chen KR ${ }^{1}$, Wang YX and Wang $Y$}

${ }^{1}$ Department of Plant Pathology, Agriculture College, Guizhou University, Guiyang, Guizhou Province, 550025, China

${ }^{2}$ Department of Crop Sciences, College of Agricultural and Marine Sciences, Sultan Qaboos University, P.O. Box 8, 123, Al Khoud, Oman

${ }^{3}$ Guizhou Light Industry Technical College, Guiyang, Guizhou Province, 550025, China

${ }^{4}$ The People's Government of Quanba Township, Yanhe County, Guizhou 565313, China

${ }^{5}$ Tea College, Guizhou University, Guiyang, Guizhou Province, 550025, China

Ran SF, Maharachchikumbura SSN, Ren YL, Liu H, Chen KR, Wang YX, Wang Y - 2017 Two new records in Pestalotiopsidaceae associated with Orchidaceae disease in Guangxi Province, China. Mycosphere 8(1), 121-130, Doi 10.5943/mycosphere/8/1/11

\begin{abstract}
Two coelomycetous taxa belonging to Pestalotiopsidaceae were collected from dried stems and disease leaves of Orchidaceae, collected from Guangxi Province, China. After morphological observation, these two taxa were found to belong to Pestalotiopsis and Neopestalotiopsis, respectively. Analysis of combined ITS, $\beta$-tubulin and tef1 gene regions indicated that these two fungal strains are Neopestalotiopsis protearum and Pestalotiopsis chamaeropsis. Based on morphological evidence and phylogenetic analysis, Neopestalotiopsis protearum and Pestalotiopsis chamaeropsis are reported from China for the first time. The taxa are described and illustrated for ease in future disease identifications.
\end{abstract}

Key words - morphology - orchid - phylogeny - taxonomy

\section{Introduction}

The genus Pestalotiopsis Steyaert was established by Steyaert (1949) and is placed in Pestalotiopsidaceae Amphisphaeriales or Xylariales (Senanayake et al. 2015). Pestalotioid fungi are commonly distributed in tropical and temperate ecosystems (Maharachchikumbura et al. 2011, 2012, Ariyawansa et al. 2015, Hyde et al. 2016) and are plant pathogens (Maharachchikumbura et al. 2013a, b), endophytes that produce a variety of bioactive secondary metabolites (Hu et al. 2007, $\mathrm{Xu}$ et al. 2010, 2014, Debbab et al. 2012, Heinig et al. 2013) and saprobes (Wu et al. 1982, Yanna et al. 2002, Hu et al. 2007, Maharachchikumbura et al. 2014a). Maharachchikumbura et al. (2014b) contributed to a major revision of Pestalotiopsis-like taxa and established two novel genera, Neopestalotiopsis and Pseudopestalotiopsis based on morphology and sequence data. Neopestalotiopsis is easily distinguished from Pseudopestalotiopsis and Pestalotiopsis by its versicoloured median cells (Maharachchikumbura et al. 2014b). Species with dark concolourous median cells with knobbed apical appendages were defined as Pseudopestalotiopsis (Maharachchikumbura et al. 2014b). Since the monograph of Maharachchikumbura et al. (2014b), 
several new pestaloid species have also been introduced (Ariyawansa et al. 2015, Hyde et al. 2016, Jayawardera et al. 2016, Maharachchikumbura et al. 2016a, b, c).

In 2015, we surveyed the orchid fungal diseases in Yachang National Nature Reserve, in Guangxi Province, China. More than 90 plant samples were collected, and after isolation in the laboratory, more than 200 strains were obtained, which included species of Alternaria, Colletotrichum, Diaporthe, Neopestalotiopsis, Pestalotiopsis and Phoma based on NCBI-BLASTs of ITS sequence data. In this paper, we report on one strain of each Pestalotiopsis and Neopestalotiopsis which are important as they cause disease of leaves of Vandopsis gigantea and diseased stems of Bulbophyllum thouars. Their taxonomic placement was evaluated by morphological comparison and combined multi-gene analyses (ITS $+\beta$-tubulin+tef1).

\section{Materials \& Methods}

\section{Isolates and morphology}

All samples were collected from Guangxi in China. The Guangxi Yachang Orchid Germplasm Gene Park, located in the Leye county, Baise City, Guangxi Zhuang Automomous Region. The Gene Park, located under the subtropical evergreen broad-leaved forest near the wind cave in the Guangxi Yachang Orchid National Nature Reserve, covers an area of over 133.3 acres, at the altitude of $985-1000 \mathrm{~m}$, with the annual average temperature of $16^{\circ} \mathrm{C}$. Strains were isolated by single spore culture technique to obtain pure colonies (Maharachchikumbura et al. 2013c). They were then transferred to potato-dextrose agar (PDA) and incubated at room temperature $\left(25^{\circ} \mathrm{C}\right)$. The morphological characters of colonies were recorded according to the reference of $\mathrm{Hu}$ et al. (2007). Conidia and conidiophores were observed using a compound microscope (Nikon Eclipse E600 DIC microscope and a Nikon DS-U2 camera or a Nikon 80i compound microscope fitted with a Canon 450D digital camera). The specimens and living culture are also deposited in the herbarium of the Department of Plant Pathology, Agricultural College, Guizhou University (HGUP).

\section{DNA extraction and sequencing}

Fresh fungal mycelia were scraped from the cultures grown on PDA medium at $18{ }^{\circ} \mathrm{C}$ until nearly covering the Petri dish. A BIOMIGA Fungus Genomic DNA Extraction Kit (GD2416) was used to rapidly extract their genome DNA. DNA amplification was performed in three regions of the internal transcribed spacers (primers ITS4 and ITS5) (White et al. 1990), partial $\beta$-tubulin gene region (primers BT2A and BT2B) (Glass \& Donaldson et al. 1995, O'Donnell \& Cigelnik et al. 1997), tef1 (primers EF1-526 F and EF1-1567R) (Rehner et al. 2001). PCR was performed with the $25 \mu \mathrm{L}$ reaction system containing $9.5 \mu \mathrm{L}$ of double distilled water, $12.5 \mu \mathrm{L}$ of $2 \times$ Taq buffer with $\mathrm{MgCl}_{2}, 1.0 \mu \mathrm{L}$ of each primer, and $1.0 \mu \mathrm{L}$ of DNA template. The thermal cycling protocols follow (Maharachchikumbura et al. 2012). Amplified PCR fragments were checked on $1 \%$ agarose electrophoresis gels stained with ethidium bromide (EB). Purified PCR products were sent to SinoGenoMax Co., Beijing, China for sequencing. Sequences generated in this study were deposited at GenBank (Table 1).

\section{Phylogenetic analyses}

Two phylogenies were used to show the placement of new isolates in Pestalotiopsis and Neopestalotiopsis with data from Maharachchikumbura et al. (2014b, 2016a, b, c), Ariyawansa et al. (2015), Hyde et al. (2016) and Jayawardera et al. (2016). Sequences were optimized manually to allow maximum alignment and maximum sequence similarity, as detailed in (Maharachchikumbura et al. 2012). A maximum parsimony analysis (MP) was performed using PAUP (Phylogenetic Analysis Using Parsimony) v. $4.0 \mathrm{~b} 10$ (Swofford 2002). Ambiguously aligned regions were excluded and gaps were treated as missing data. Trees were inferred using the heuristic search option with TBR branch swapping and 1,000 random sequence additions. Maxtrees were set up to 5,000 , branches of zero length were collapsed and all multiple parsimonious trees were saved. Tree length [TL], consistency index $[\mathrm{CI}]$, retention index [RI], rescaled consistency index [RC], homoplasy index $[\mathrm{HI}]$, and log likelihood [-ln L] (HKY model) were calculated for trees generated 
under different optimality criteria. The robustness of the most parsimonious trees was evaluated by 1,000 bootstrap replications resulting from maximum parsimony analysis, each with 10 replicates of random stemwise addition of taxa (Felsenstein 1985). Trees were viewed in Treeview (Page 1996). Sequences were generated from forward and reverse primers and these were subsequently lodged with GenBank (Table 1).

Table 1 Sequences used for phylogenetic analysis. Most sequences are from Maharachchikumbura et al. (2014) and Jayawardera et al. (2016).

\begin{tabular}{|c|c|c|c|c|}
\hline \multirow{2}{*}{ Taxon } & \multirow{2}{*}{ Isolate number } & \multicolumn{3}{|c|}{ GenBank accession Number } \\
\hline & & ITS & $\beta$-tubulin & tef1 \\
\hline Neopestalotiopsis aotearoa & CBS 367.54 & KM199369 & KM199454 & KM199526 \\
\hline N. asiatica & MFLUCC12-0286 & JX398983 & JX399018 & JX399049 \\
\hline N. australis & CBS 114159 & KM199348 & KM199432 & KM199537 \\
\hline N. chrysea & MFLUCC12-0261 & JX398985 & JX399020 & JX399051 \\
\hline N. chrysea & MFLUCC 12-0262 & JX398986 & JX399021 & JX399052 \\
\hline N. clavispora & MFLUCC 12-0280 & JX398978 & JX399013 & JX399044 \\
\hline N. clavispora & MFLUCC 12-0281 & JX398979 & JX399014 & JX399045 \\
\hline N. cubana & CBS 600.96 & KM199347 & KM199438 & KM199521 \\
\hline N. ellipsospora & CBS 115113 & KM199343 & KM199450 & KM199544 \\
\hline N. ellipsospora & MFLUCC 12-0283 & JX398980 & JX399016 & JX399047 \\
\hline N. eucalypticola & CBS 264.37 & KM199376 & KM199431 & KM199551 \\
\hline N. formicarum & CBS 115.83 & KM199344 & KM199444 & KM199519 \\
\hline N. formicarum & CBS 362.72 & KM199358 & KM199455 & KM199517 \\
\hline N. honoluluana & CBS 111535 & KM199363 & KM199461 & KM199546 \\
\hline N. honoluluana & CBS 114495 & KM199364 & KM199457 & KM199548 \\
\hline N. javaensis & CBS 257.31 & KM199357 & KM199437 & KM199543 \\
\hline N. protearum & CBS 114178 & JN712498 & KM199463 & KM199542 \\
\hline N. protearum & HGUP7003 & KX196813 & KX673488 & KX673490 \\
\hline N. rosae & CBS 101057 & KM199359 & KM199429 & KM199523 \\
\hline N. rosae & CBS 124745 & KM199360 & KM199430 & KM199524 \\
\hline N. samarangensis & CBS 115451 & KM199365 & KM199447 & KM199556 \\
\hline N. samarangensis & SS010 & JQ968609 & JQ968610 & JQ968611 \\
\hline N. saprophytica & CBS 115452 & KM199345 & KM199433 & KM199538 \\
\hline N. saprophyta & MFLUCC 12-0282 & JX398982 & JX399017 & JX399048 \\
\hline N. surinamensis & CBS 111494 & - & KM199462 & KM199530 \\
\hline N. surinamensis & CBS 450.74 & KM199351 & KM199465 & KM199518 \\
\hline N. umbrinospora & MFLUCC 12-0285 & JX398984 & JX399019 & JX399050 \\
\hline N. vitis & CBS 110.20 & KM199342 & KM199442 & KM199540 \\
\hline N. zimbabwana & CBS 111495 & - & KM199456 & KM199545 \\
\hline Neopestalotiopsis sp. & CBS 233.79 & KM199373 & KM199464 & KM199528 \\
\hline Neopestalotiopsis sp. & CBS 322.76 & KM199366 & KM199446 & KM199536 \\
\hline Neopestalotiopsis sp. & CBS 664.94 & KM199354 & KM199449 & KM199525 \\
\hline Neopestalotiopsis sp. & CBS 164.42 & KM199367 & KM199434 & KM199520 \\
\hline Neopestalotiopsis sp. & CBS 360.61 & KM199346 & KM199440 & KM199522 \\
\hline Neopestalotiopsis sp. & CBS 119.75 & KM199356 & KM199439 & KM199531 \\
\hline Neopestalotiopsis sp. & CBS 266.80 & KM199352 & - & KM199532 \\
\hline Neopestalotiopsis sp. & CBS 266.37 & KM199349 & KM199459 & KM199547 \\
\hline Neopestalotiopsis sp. & CBS 323.76 & KM199350 & KM199458 & KM199550 \\
\hline Neopestalotiopsis sp. & CBS 361.61 & KM199355 & KM199460 & KM199549 \\
\hline Pestalotiopsis adusta & ICMP 6088 & JX399006 & JX399037 & JX399070 \\
\hline P. australis & CBS 111503 & KM199331 & KM199382 & KM199557 \\
\hline P. australis & CBS 114193 & KM199332 & KM199383 & KM199475 \\
\hline P. australis & CBS 114474 & KM199334 & KM199385 & KM199477 \\
\hline P. australis & CBS 119350 & KM199333 & KM199384 & KM199476 \\
\hline P. biciliata & CBS 124463 & KM199308 & KM199399 & KM199505 \\
\hline
\end{tabular}




\begin{tabular}{|c|c|c|c|c|}
\hline P. chamaeropis & CBS 113604 & KM199323 & KM199389 & KM199471 \\
\hline P. chamaeropis & CBS 113607 & KM199325 & KM199390 & KM199472 \\
\hline P. chamaeropis & CBS 186.71 & KM199326 & KM199391 & KM199473 \\
\hline P. chamaeropis & CBS 237.38 & KM199324 & KM199392 & KM199474 \\
\hline P. chamaeropis & HGUP7002 & KX196814 & KX673487 & KX673489 \\
\hline P. clavata & MFLUCC 12-0268 & JX398990 & JX399025 & JX399056 \\
\hline P. furcata & MFLUCC 12-0054 & JQ683724 & JQ683708 & JQ683740 \\
\hline P. hollandica & CBS 265.33 & KM199328 & KM199388 & KM199481 \\
\hline P. humus & CBS 336.97 & KM199317 & KM199420 & KM199484 \\
\hline P. inflexa & MFLUCC 12-0270 & JX399008 & JX399039 & JX399072 \\
\hline P. intermedia & MFLUCC 12-0259 & JX398993 & JX399028 & JX399059 \\
\hline P. knightiae & CBS 114138 & KM199310 & KM199408 & KM199497 \\
\hline P. linearis & MFLUCC 12-0271 & JX398992 & JX399027 & JX399058 \\
\hline P. monochaeta & CBS 144.97 & KM199327 & KM199386 & KM199479 \\
\hline P. monochaeta & CBS 440.83 & KM199329 & KM199387 & KM199480 \\
\hline P. oryzae & CBS 353.69 & KM199299 & KM199398 & KM199496 \\
\hline P. papuana & CBS 887.96 & KM199318 & KM199415 & KM199492 \\
\hline$P$. portugalica & CBS 393.48 & KM199335 & KM199422 & KM199510 \\
\hline P. rhododendri & OP086 & KC537804 & KC537818 & KC537811 \\
\hline P. rosea & MFLUCC 12-0258 & JX399005 & JX399036 & JX399069 \\
\hline P. scoparia & CBS 176.25 & KM199330 & KM199393 & KM199478 \\
\hline P. unicolor & MFLUCC 12-0275 & JX398998 & JX399029 & JX399063 \\
\hline P. unicolor & MFLUCC 12-0276 & JX398999 & JX399030 & - \\
\hline
\end{tabular}

\section{Results}

\section{Phylogenetic analyses}

The alignment for Neopestalotiopsis comprised 1845 characters including gaps (ITS: 1-547, B-tubulin: 548-1308 and tef1: 1309-1845). Among these characters, 1348 were constant, 223 variable characters were parsimony-uninformative and 274 are parsimony-informative. Tree Length $=887, \mathrm{CI}=0.71, \mathrm{RI}=0.79, \mathrm{RC}=0.60$ and $\mathrm{HI}=0.29$. The parsimony analysis resulted in 150 equally parsimonious trees and the first tree represent in Fig. 1. In the Neopestalotiopsis tree (Fig. 1), all Neopestalotiopsis isolates and Pseudopestalotiopsis isolates grouped into two clades, respectively. our strain HGUP 7003 clustered together with Neopestalotiopsis protearum (CBS 114178), Neopestalotiopsis sp. (CBS 233.79) and N. surinamensis (CBS 450.74).

The alignment for Pestalotiopsis consisted of 77 taxa, including Neopestalotiopsis saprophyta (MFLUCC 12-0282) as the outgroup taxon. The alignment comprised with 2007 characters including gaps (ITS: 1-557, $\beta$-tubulin: 558-1446 and tef1: 2447-2007). Among them, 1334 characters are constant, 297 variable characters are parsimony-uninformative and 376 are parsimony-informative. The parsimony analysis resulted in 21 equally parsimonious trees and the first tree ( $\mathrm{TL}=1507, \mathrm{CI}=0.62, \mathrm{RI}=0.81, \mathrm{RC}=0.50$ and $\mathrm{HI}=0.38$ ) was selected to represented as Fig. 2. In Fig. 2, our isolate HGUP 7002 clusters with four P. chamaeropis isolate (CBS 113604, CBS 113607, CBS 186.71 and CBS 237.38) with 68\% bootstrap support. 


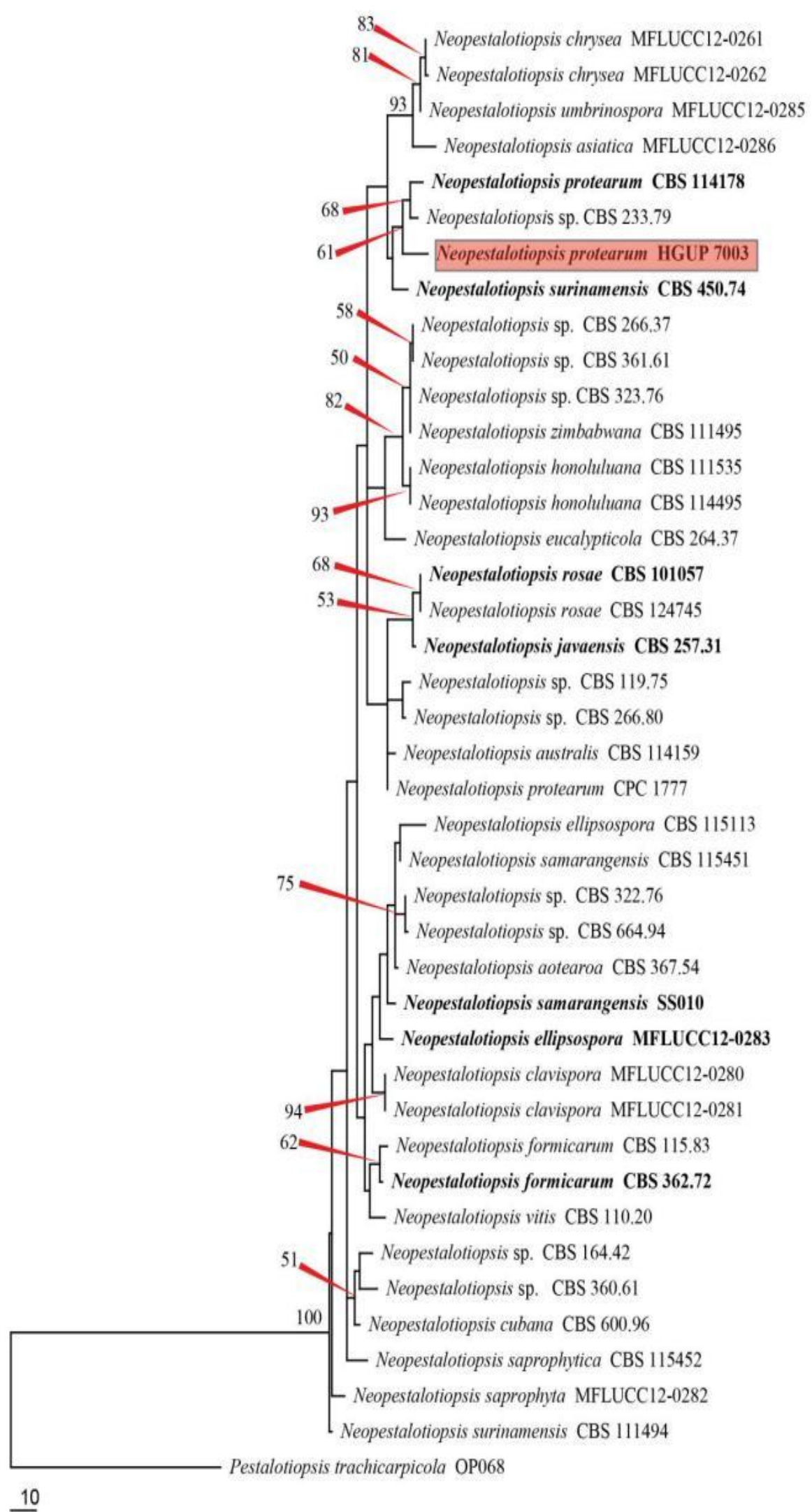

Figure 1 - Phylogenetic tree for Neopestalotiopsis based on maximum parsimony (MP) generated from combination of ITS, $\beta$-tubulin and tef 1 sequences. Pestalotiopsis trachicarpicola (OP068) was used as the outgroup taxon. New strains are in bold and red and MP bootstrap values higher than $50 \%$ are shown at nodes. Type or voucher specimens are in bold. 


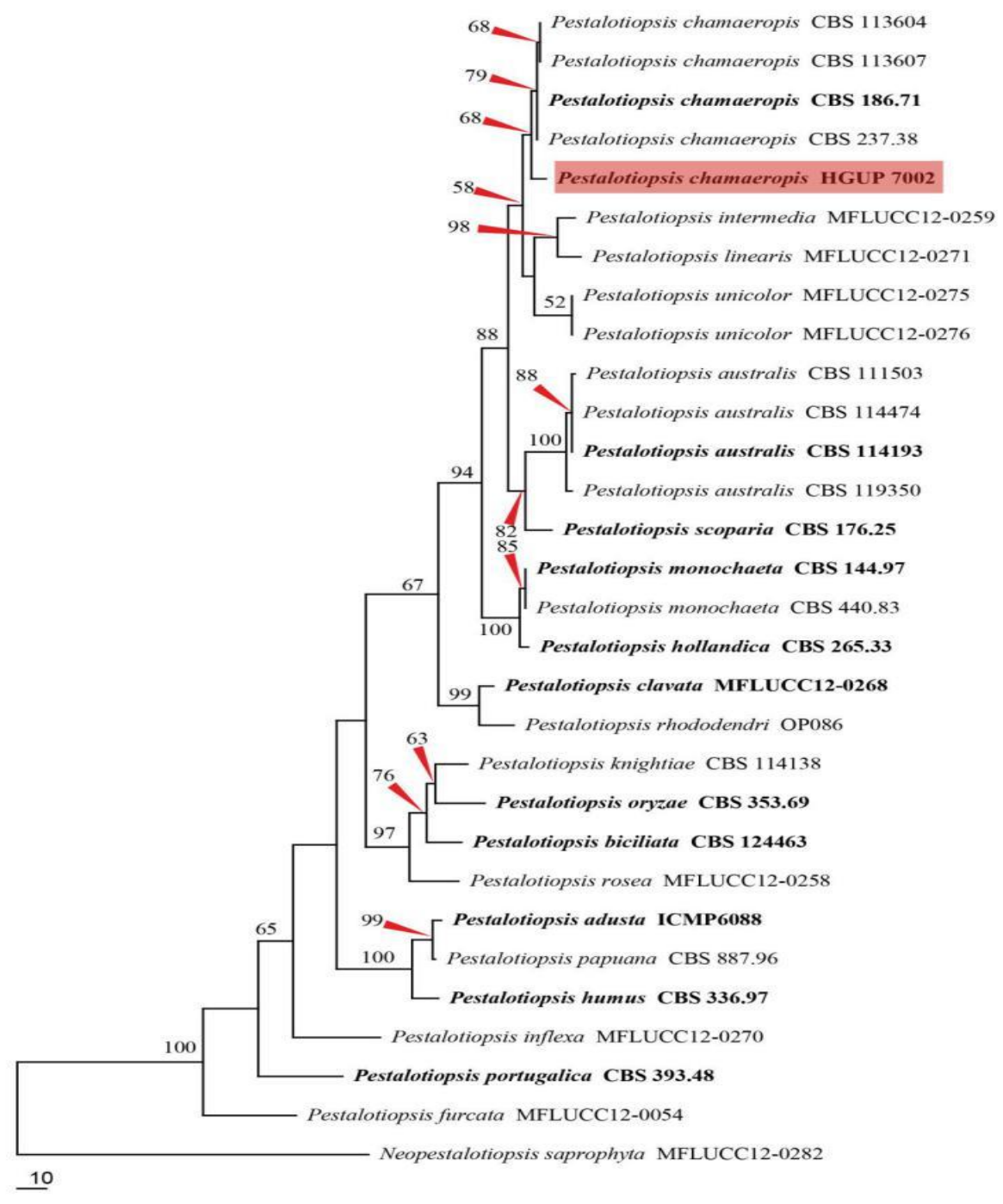

Figure 2 - Phylogenetic tree for Pestalotiopsis based on maximum parsimony generated from combination of ITS, $\beta$-tubulin and tef1 sequences. Neopestalotiopsis saprophyta (MFLUCC 120282) was used as the outgroup taxon. New strains are in bold and red and MP bootstrap values higher than $50 \%$ are shown at nodes. Type or voucher specimens are in bold

\section{Taxonomy}

Neopestalotiopsis protearum (Crous \& L. Swart) Maharachch., K.D. Hyde \& Crous, Studies in Mycology 79: 147 (2014b) Fig. 3

三Pestalotiopsis protearum Crous \& L. Swart, Persoonia 27: 34 (2011)

Material examined - China, Guangxi, Baise City, Yachang Orchid National Nature Reserve, on dead stem of Bulbophyllum thouars (Orchidaceae), 9 November 2015, S.F. Ran (HGUP 7003), living culture YC5502.

On dead stem of orchid. Sexual morph: Undetermined. Asexual morph: Coelomycetous. Conidiomata pycnidial, globose, solitary, black. Conidiophores 3.8-16.3 $\times 1.7-2.5 \mu \mathrm{m}(\overline{\mathrm{x}}=8.1 \times$ $2.2 \mu \mathrm{m}, \mathrm{n}=40$ ) long, cylindrical, hyaline to subhyaline, smooth-walled. Conidiogenous cells simple, integrated, hyaline. Conidia fusiform, straight to slightly curved, 4 septate, 18.3-25.5 $\times$ 5.7-8.1 $\mu \mathrm{m}(\overline{\mathrm{x}}=22.0 \times 7.0 \mu \mathrm{m}, \mathrm{n}=40)$, three median cells versicolourous, doliiform to cylindrical, $14.1-17.3 \times 5.7-8.1 \mu \mathrm{m}(\overline{\mathrm{x}}=15.5 \times 7.0 \mu \mathrm{m}, \mathrm{n}=40)$, constricted at the septa; apical cell hyaline, obconic to cylindrical, $2.0-4.5 \mu \mathrm{m}(\overline{\mathrm{x}}=3.1 \mu \mathrm{m}, \mathrm{n}=40)$ long, 2.6-4.5 $\mu \mathrm{m}(\overline{\mathrm{x}}=3.6 \mu \mathrm{m}, \mathrm{n}=40)$ width, with 1 to 3 (often 3 ) tubulars apical appendage, arising from the apical crest, $12-22 \mu \mathrm{m},(\overline{\mathrm{x}}=$ $17.7 \mu \mathrm{m}, \mathrm{n}=40)$; basal cell obconic to cylindrical, hyaline, $3.2-5.2 \mu \mathrm{m}(\overline{\mathrm{x}}=3.85 \mu \mathrm{m}, \mathrm{n}=40)$ 
length, 3-4.3 $\mu \mathrm{m}(\overline{\mathrm{x}}=3.7 \mu \mathrm{m}, \mathrm{n}=40)$ width, basal appendages filiform, unbranched, 1.6-3.8 $\mu \mathrm{m}(\overline{\mathrm{x}}$ $=2.7 \mu \mathrm{m}, \mathrm{n}=40$ ) long.

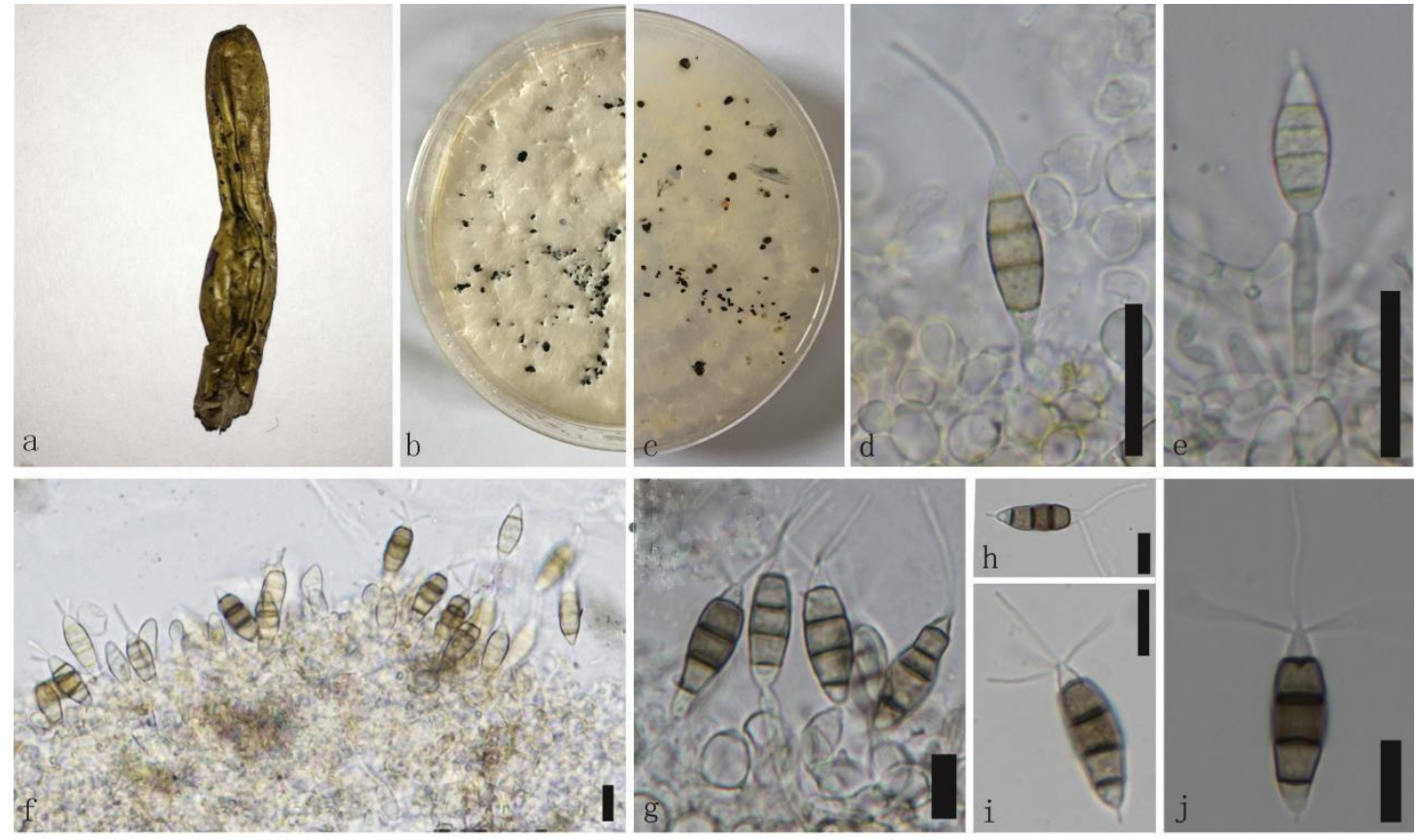

Figure 3 - Neopestalotiopsis protearum (HGUP 7003). a Host material. b Colony on PDA from above. c. from below. $\mathbf{d}-\mathbf{g}$ Conidioma and conidiophores. $\mathbf{h}-\mathbf{j}$ Conidia. Scale bars: $\mathbf{d}-\mathbf{e}=20 \mu \mathrm{m}$, $\mathbf{f}-\mathbf{h}=5 \mu \mathrm{m}, \mathbf{i}-\mathbf{j}=10 \mu \mathrm{m}$.

Pestalotiopsis chamaeropis Maharachch., K.D. Hyde \& Crous, Studies in Mycology 79: 158 (2014)

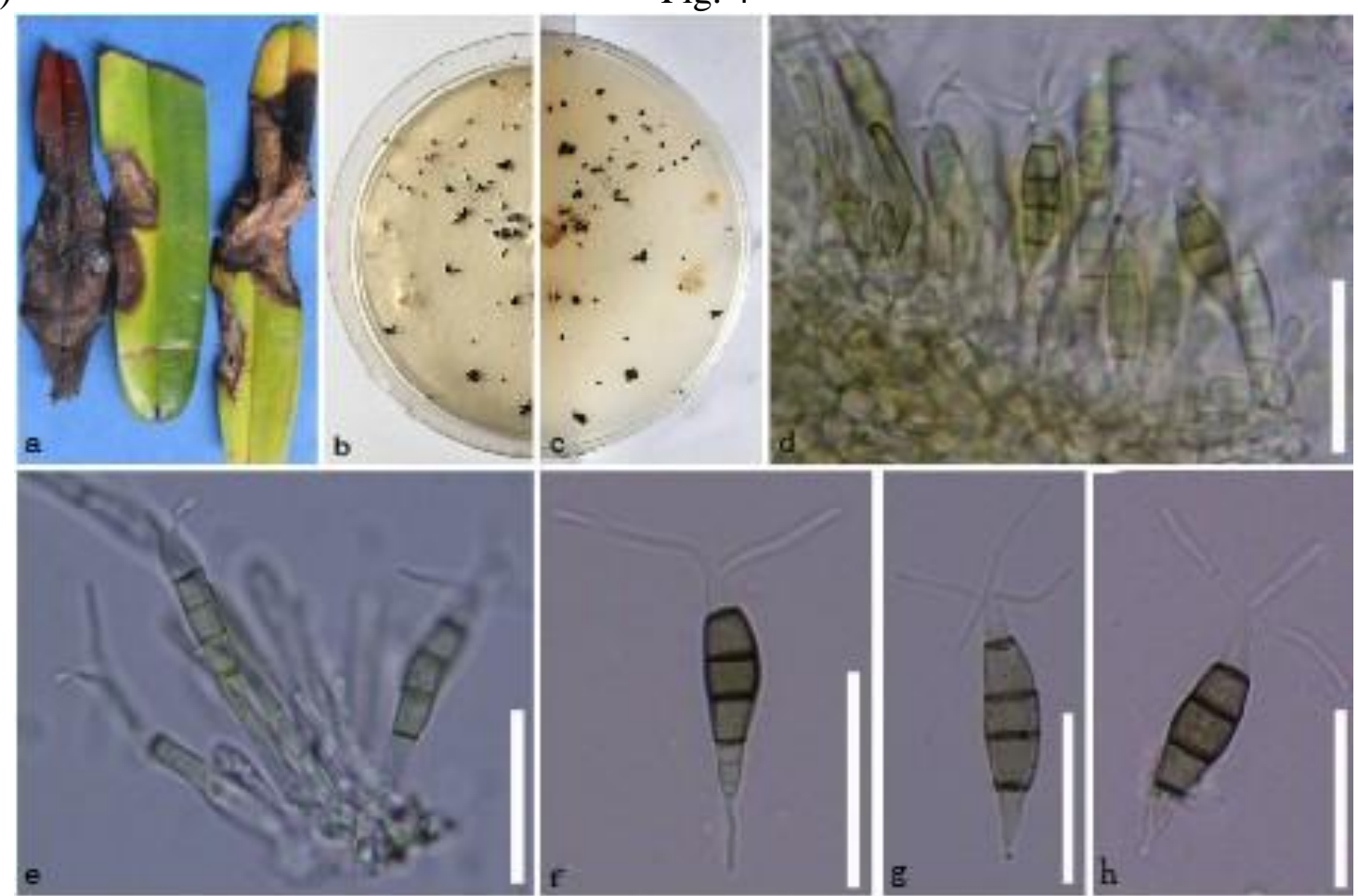

Fig 4 - Pestalotiopsis chamaeropis (HGUP 7002). a Diseased host material. b Colony on PDA from above. c. Colony on PDA from below. $\mathbf{d}-\mathbf{e}$ Conidioma and conidiophores. $\mathbf{f}-\mathbf{h}$ Conidia. Scale bars: $\mathbf{a}-\mathbf{h}=10 \mu \mathrm{m}$. 
Material examined - China, Guangxi, Baise City, Yachang Orchid National Nature Reserve, on dead stem of Vandopsis gigantea (Orchidaceae), 9 November 2015, S.F. Ran (HGUP 7002), living culture YC5582.

Habit Vandopsis gigantea. Conidiomata pycnidial, globose, solitary, black. Conidiophores 4.7-23.2 × 2.7-5.1 $\mu \mathrm{m}(\overline{\mathrm{x}}=12.6 \times 4.0 \mu \mathrm{m}, \mathrm{n}=40)$ long, subcylindrical, hyaline, verruculose. Conidiogenous cells simple, integrated, hyaline. Conidia fusiform, straight to slightly curved, 4 septate, $21.2-26.3 \times 5.4-6.7 \mu \mathrm{m}(\overline{\mathrm{x}}=23.9 \times 6.1 \mu \mathrm{m}, \mathrm{n}=40)$; three median cell concolourous, , smooth-walled, doliiform to cylindrical, brown, 13.3-16.8 $\times 5.4-6.7 \mu \mathrm{m}(\overline{\mathrm{x}}=15.2 \times 6.1 \mu \mathrm{m}, \mathrm{n}=$ 40); apical cell hyaline, subcylindrical, $4.3-5.3 \mu \mathrm{m}(\overline{\mathrm{x}}=4.6 \mu \mathrm{m}, \mathrm{n}=40)$, with 1 to 4 tubulars apical appendage, arising from the apical crest, $11-24 \mu \mathrm{m}(\overline{\mathrm{x}}=16.3 \mu \mathrm{m}, \mathrm{n}=40)$; basal cell obconic to cylindrical, hyaline, 3-5.9 $\times 2.8-4.6 \mu \mathrm{m}(\overline{\mathrm{x}}=4.4 \times 3.5 \mu \mathrm{m}, \mathrm{n}=40)$, basal appendage filiform, unbranched, $2.6-7.8 \mu \mathrm{m}(\overline{\mathrm{x}}=5.1 \mu \mathrm{m}, \mathrm{n}=40)$ long.

\section{Discussion}

Neopestalotiopsis protearum collected in this study have conidia $(26-28.5 \times 7-7.5 \mu \mathrm{m})$ which are similar in size to those of $N$. surinamensis $(24-28 \times 7.5-9 \mu \mathrm{m})$ (Crous et al. 2011, Maharachchikumbura et al. 2014b) and overlap with those of the type of $N$. protearum. The molecular analysis (Fig. 1) however, indicates that our collection is more similar to N. protearum. The collection of Pestalotiopsis chamaeropis is similar to the type of $P$. chamaeropis and this is confirmed by the sequence data. The MP bootstrap support for naming our new collections as Neopestalotiopsis protearum (61\%) and Pestalotiopsis chamaeropis (68\%) is relatively low. This is probably because of the short $\beta$-tubulin and tef 1 sequences of the isolates introduced in present study. In view of the phylogenetic analysis and morphological comparison, we conclude that our two isolates represent two Chinese new records in Pestalotiopsidaceae, Neopestalotiopsis protearum and Pestalotiopsis

\section{Acknowledgements}

The research is supported by the projects of National Natural Science Foundation of China (No. 31560489), Fundamental Research on Science and Technology, Ministry of Science and Technology of China (2014FY120100), Qiandongnan of Guizhou Province, Blueberry industry science of Technology cooperation (No. [2015]06), Guizhou Province, medicinal plant breeding and cultivation of talent base (No. [2013]15) and postgraduate education innovation program of Guizhou Province (ZYRC [2014]004).

\section{References}

Ariyawansa HA, Kevin D. Hyde, Subashini C, et al. 2015 - Fungal diversity notes 111-252taxonomic and phylogenetic contributions to fungal taxa. Fungal Diversity 75, 27-274.

Crous PW, Summerell BA, Swart L, Denman S, et al. 2011 - Fungal pathogens of Proteaceae. Persoonia 27, 20-45.

Debbab A, Aly AH, Proksch P. 2012 - Endophytes and associated marine derived fungiecological and chemical perspectives. Fungal Diversity 57, 45-83.

Felsenstein J. 1985 -Confidence limits on phylogenies: an approach using the bootstrap. Evolution 39, 783-791.

Glass NL, Donaldson GC. 1995 - Development of primer sets designed for use with the PCR to amplify conserved genes from filamentous ascomycetes. Applied and Environmental Microbiology 61, 1323-1330.

Heinig U, Scholz S, Jennewein S. 2013 - Getting to the bottom of taxol biosynthesis by fungi Fungal Diversity 60, 161-170.

Hu HL, Jeewon R, Zhou DQ, Zhou TX, Hyde KD. 2007 - Phylogenetic diversity of endophytic Pestalotiopsis species in Pinus armandii and Ribes spp.: evidence from rDNA and $\beta$-tubulin gene phylogenies. Fungal Diversity 24, 1-22. 
Hyde KD, Hongsanan S, Jeewon R, et al. 2016 - Fungal diversity notes 367-490-taxonomic and phylogenetic contributions to fungal taxa. Fungal Diversity 80, 1-270.

Maharachchikumbura SSN, Guo LD, Chukeatirote E, Bahkali AH, Hyde KD. 2011 Pestalotiopsis-morphology, phylogeny, biochemistry and diversity, Fungal Diversity 50, 167-187.

Maharachchikumbura SSN, Guo LD, Cai L, et al. 2012 - A multi-locus backbone tree for Pestalotiopsis, with a polyphasic characterization of 14 new species. Fungal Diversity 56, 95-129.

Maharachchikumbura SSN, Guo LD, Chukeatirote E, McKenzie EHC, Hyde KD. 2013a - A destructive new disease of Syzygium samarangense in Thailand caused by the new species Pestalotiopsis samarangensis. Tropical Plant Pathology 38, 227-235.

Maharachchikumbura SSN, Zhang YM, Wang Y, Hyde KD. 2013b - Pestalotiopsis anacardiacearum sp. nov. (Amphisphaeriaceae) has an intricate relationship with Penicillaria jocosatrix, the mango tip borer. Phytotaxa 99, 49-57.

Maharachchikumbura SSN, Chukeatirote E, Guo LD, Crous PW et al. 2013c - Pestalotiopsis species associated with Camellia sinensis (tea). Mycotaxon 123, 47-61.

Maharachchikumbura SSN, Guo LD, Chukeatirote E, Hyde KD. 2014a - Improving the backbone tree for the genus Pestalotiopsis ; addition of P. steyaertii and P. magna sp. nov. Mycological Progress 13, 617-624.

Maharachchikumbura SSN, Hyde KD, Groenewald JZ, Xu J, Crous PW. 2014b - Pestalotiopsis revisited. Studies in Mycology 79, 121-186.

Maharachchikumbura SSN, Guo LD, Liu ZY, Hyde KD. 2016a - Pseudopestalotiopsis ignota and Ps. camelliae spp. nov. associated with grey blight disease of tea in China. Mycological Progress 15, 1-7.

Maharachchikumbura SSN, Larignon P, Hyde KD, Al-Sadi AM, Liu ZY. 2016b - Characterization of Neopestalotiopsis, Pestalotiopsis and Truncatella species associated with grapevine trunk diseases in France. Phytopathologia Mediterranea 55, 380-390.

Maharachchikumbura SSN, Wu SP, Hyde KD, Al-Sadi AM, Liu ZY. 2016c - First report of sweet potato leaf spot caused by Neopestalotiopsis ellipsospora in Guizhou province, China. Journal of Plant Pathology 98, 686.

O’Donnell K, Cigelnik E. 1997 - Two divergent intragenomic rDNA ITS2 types within a monophyletic lineage of the fungus Fusarium are nonorthologous. Molecular Phylogenetics and Evolution 7, 103-116.

Page RD. 1996 - TreeView: an application to display phylogenetic trees on personal computers. Computer Applications in the Biosciences 12, 357-358.

Rehner S. 2001 - Primers for elongation factor 1- $\alpha$ (EF1- $\alpha)$. http://ocid. nacas.org/ research/ deephyphae/ EF1 primer.pdf.

Jayawardene RS, Liu M, Maharachchikumbura SSN, et al. 2016 - Neopestalotiopsis vitis sp. nov. causing grapevine leaf spot in China. Phytotaxa 258, 63-74.

Senanayake IC, Maharachchikumbura SSN, Hyde KD, et al. 2015 - Towards unraveling relationships in Xylariomycetidae (Sordariomycetes ). Fungal Diversity 73, 1-72.

Steyaert RL. 1949 - Contribution a l'etude monographique de Not. Et Monochaetia Sacc, (Truncatella gen. nov. et Pestalotiopsis gen. nov.). Bulletin Du Jardin Botanique De L État A Bruxelles 19, 285-354.

Swofford DL. 2002 - PAUP*: Phylogenetic Analysis Using Parsimony and other methods, version 4.0 b10. Sunderland, MA: Sinauer Associates.

White TJ, Bruns T, Lee S, Taylor J. 1990 - Amplification and direct sequencing of fungal ribosomal RNA genes for phylogenetics. In: PCR Protocols: a guide to methods and applications (Innis MA, Gelfand DH, Sninsky JJ, White TJ, eds). Academic Press, San Diego, California, USA, 315-322.

Wu CG, Tseng HY, Chen ZC. 1982 - Fungi inhabiting on Schoenoplectus triqueter (L.) Palla (I). Taiwania 27, 35-38. 
Xu J, Ebada SS, Proksch P. 2010 - Pestalotiopsis a highly creative genus: Chemistry and bioactivity of secondary metabolites. Fungal Diversity 44, 15-31.

$\mathrm{Xu}$ J, Yang X, Lin Q. 2014 - Chemistry and biology of Pestalotiopsis-derived natural products. Fungal Diversity 54, 956-987.

Yanna, Ho WH, Hyde KD. 2002 - Fungal succession on fronds of Phoenix hanceana in Hong Kong. Fungal Diversity 10, 185-211. 